\title{
Caracterización de las personas que demandaron la prueba de detección rápida del VIH en Andalucía entre 2013 y 2014
}

\section{Characterising people who demanded the rapid HIV test in Andalusia between 2013 and 2014}

doi.org/10.23938/ASSN.0010

\author{
N. Lorusso', F. Lozano ${ }^{2}$, C. Zamora ${ }^{3}$
}

\section{RESUMEN}

Fundamento. El objetivo del estudio fue caracterizar a las personas que demandaron la prueba rápida VIH en Andalucía.

Métodos. Estudio trasversal realizado por las entidades sociales colaboradoras del Programa de diagnóstico precoz del VIH en Andalucía. Los participantes fueron personas que demandaron la prueba en fluido oral y que contestaron una encuesta previa a la misma entre septiembre de 2013 y agosto de 2014. Se realizó un análisis descriptivo y de regresión logística para determinar los factores de riesgo asociados a un resultado reactivo. Se analizaron variables relacionadas con: entidad que realizó la prueba, características sociodemográficas, exposición de riesgo, antecedentes de realización de la prueba $\mathrm{VIH}$, antecedentes de infecciones de transmisión sexual (variables predictivas) y resultado de la prueba (variable dependiente)

Resultados. Las personas caracterizadas fueron 1.844 (48\%), en su mayoría hombres (70\%) que practicaron sexo con otros hombres (HSH) en los últimos 12 meses (59\%). El $53 \%$ se había realizado la prueba anteriormente y acudieron tras una exposición de riesgo, principalmente de sexo desprotegido oral ( $75 \%$ ), vaginal (50\%) y/o anal (38\%). El $23 \%$ fueron extranjeros. Los factores asociados a un resultado reactivo fueron: entidades especializadas en $\mathrm{VIH}$, sexo masculino, HSH, realización de sexo desprotegido (anal, con persona $\mathrm{VIH}+\mathrm{y} / \mathrm{o}$ con trabajador/a del sexo) y tener antecedente de prueba $(\mathrm{p}<0,05)$. La significación estadística del sexo masculino y de las prácticas desprotegidas se mantuvo en el estudio multivariante.

Conclusiones. Se ha logrado caracterizar a un elevado número de personas (alrededor del 50\%), lo que favorecerá la adecuación de la recogida de información y la mejora del Programa.

Palabras clave. Diagnóstico precoz. VIH-1. Organizaciones No Gubernamentales. Conducta Sexual. Homosexualidad.

\section{An. Sist. Sanit. Navar. 2017; 40 (1): 93-102}

1. Servicio de Epidemiología. Consejería de Sanidad. Junta de Castilla y León.

2. Unidad Clínica de Enfermedades Infecciosas y Microbiología. Hospital Universitario de Valme. Sevilla.

3. Consejería de Salud. Junta de Andalucía.

Recepción: 11/11/2016

Aceptación provisional: 27/12/2016

Aceptación definitiva: 16/02/2016

\begin{abstract}
Background. The aim of the study was to describe people who demanded the HIV screening test in Andalusia (Spain).

Design. Cross-sectional study carried out by social organisation collaborating with the Program for early diagnosis of HIV in Andalusia. Participants underwent an oral HIV test and answered a survey beforehand, from September 2013 to August 2014. The study included a descriptive analysis of data and a logistic regression in order to determine the risk factors associated with a reactive test result. We analysed variables related to: social organisation, sociodemographic characteristics, risk exposure that caused the test, previous test history, history of sexually transmitted infections (predictive variables) and test result (dependent variable).
\end{abstract}

Results. It was possible to characterise 1,844 people (48\%). They were mostly men (70\%) who had sex with men (MSM) in the last 12 months (59\%). The $53 \%$ had a previous HIV test and came to social organisations after an exposure to risk, especially unprotected oral sex (75\%), vaginal (50\%) and/or anal (38\%). Twenty-three percent was foreigners. A positive result was associated to: HIV work area, male sex, MSM, unprotected sex (anal, with HIV+ person or with sex worker) and test antecedent $(\mathrm{p}<0.05)$. These unprotected practices and male sex were also significant in the multivariate analysis.

Conclusions. This approach of the study made it possible to characterise a great number of people (around 50\%). These results will guide improvements in the Program's data collection and future community interventions.

Keywords. Early diagnosis. HIV-1. NGO. Sexual behaviour. Homosexuality. 


\section{INTRODUCCIÓN}

El diagnóstico precoz de la infección por VIH representa uno de los retos fundamentales para la salud pública. En España, se estima que el $30 \%$ de las personas con VIH desconoce su estado acerca de la infección y, asumiendo las hipótesis más conservadoras, podían ser el origen de más de la mitad de las nuevas infecciones al estar transmitiendo el virus de forma inadvertida $^{1-2}$. Para el 2014, se estimó una tasa de incidencia de 9,3 por 100.000 habitantes ajustada por retraso de notificación (por encima de la media de la UE/EEA de 6,4 por 100.000). El 46\% presentó un diagnóstico tardío (DT, recuento de $\mathrm{CD} 4 / \mu \mathrm{L}$ inferior a 350 ) y el $28 \%$ enfermedad avanzada (EA, recuento de $\mathrm{CD} 4 / \mu \mathrm{L}$ inferior a 200 ), que conlleva un retraso del inicio del tratamiento antirretroviral (TAR) con un aumento de morbilidad y mortalidad a nivel individual, un mayor coste en la terapia y en la asistencia global de la persona infectada ${ }^{3}$. En Andalucía, en el año 2014, el Registro Andaluz de Vigilancia VIH/Sida (siVIhDA), estimó una tasa de 6,9 por 100.000 habitantes no corregidos por retraso de la notificación. El 51\% de los casos presentaron DT, siendo del $30 \%$ el porcentaje de $\mathrm{EA}^{4}$.

El Programa de diagnóstico precoz del VIH de la Consejería de Salud de Andalucía, iniciado a finales de 2008, representa en la actualidad el eje de la Estrategia 4 de Mejora de la atención sanitaria y el diagnóstico precoz de VIH del Plan Andaluz frente al VIH/Sida y otras ITS 2010-2015 5 . Para su realización utiliza el Oral Quick Advance rapid HIV $1 / 2 \AA$, que detecta VIH-1/2 en fluido oral, siendo una prueba altamente sensible y específica, que proporciona un resultado mediante la lectura visual del mismo en un lapso de alrededor de 15 minutos $^{6-7}$. Esta prueba obvia la necesidad de extraer una muestra de sangre y de acudir a laboratorios especializados para su procesamiento $^{8}$. El Programa, que se puso en marcha en colaboración con las entidades sociales (asociaciones, fundaciones, organizaciones sin ánimo de lucro...), recoge las recomendaciones de los principales organismos internacionales ${ }^{9-10}$, y propugna el acercamien- to de la prueba a toda la población a través de estrategias de baja exigencia, haciendo especial énfasis en los grupos más vulnerables. La prueba es gratuita y voluntaria, garantizándose plena confidencialidad en todo momento ${ }^{11-12}$. El objetivo del estudio fue caracterizar y analizar los factores asociados a tener un resultado reactivo en las personas que demandaron, durante 12 meses consecutivos, la prueba de detección precoz del VIH en las entidades colaboradoras del Programa, con la finalidad de aportar evidencia sobre la población diana a la que se está dirigiendo y adecuar la recogida de datos para conseguir una mejor eficiencia del mismo.

\section{MATERIAL Y MÉTODOS}

Se constituyó un equipo multidisciplinar formado por profesionales del ámbito sanitario y social. Se contactó con todos los centros de las entidades colaboradoras del Programa, se expusieron los objetivos del trabajo y se solicitó colaboración voluntaria. Se obtuvo la disponibilidad explícita de colaboración por parte de 42 de las 50 entidades que participaban en el Programa durante el periodo de estudio. Se analizó la información contenida en los modelos de encuesta utilizados por cada uno de los centros, 26 utilizaban exclusivamente un modelo de encuesta básico con simples datos de actividad y 7 , un modelo propio sin interés epidemiológico que, por estos motivos, se excluyeron. Finalmente, se seleccionaron 9 centros, 6 de los cuales utilizaban más de un modelo de encuesta y 7 utilizaban el modelo de encuesta del proyecto europeo Coba-Test. Entre los centros seleccionados, 4 se dedicaban fundamentalmente a tareas relacionadas con $\mathrm{VIH} /$ sida (Tabla 1).

Tras la aprobación del Comité coordinador de ética de la investigación biomédica de Andalucía, se realizó un estudio trasversal dirigido a toda la población que demandó la prueba de detección rápida en saliva de VIH entre septiembre 2013 y agosto 2014 inclusive. La población de estudio fueron todas las personas que realizaron 
Tabla 1. Provincia, ámbito de actuación y pruebas de las entidades colaboradoras del Programa de diagnóstico precoz del VIH incluidas en el estudio. Periodo: septiembre 2013-agosto 2014

\begin{tabular}{|c|c|c|c|c|c|c|c|c|c|c|}
\hline \multirow[t]{2}{*}{ Provincia } & \multirow[t]{2}{*}{ Entidad } & \multirow{2}{*}{$\begin{array}{l}\text { Ámbito de } \\
\text { actuación }\end{array}$} & \multicolumn{2}{|c|}{ Reactivos } & \multicolumn{2}{|c|}{ No Reactivos } & \multicolumn{2}{|c|}{$\begin{array}{l}\text { Resultados } \\
\text { Perdidos }\end{array}$} & \multicolumn{2}{|c|}{$\begin{array}{l}\text { Total de } \\
\text { Pruebas }\end{array}$} \\
\hline & & & No & $\%$ & № & $\%$ & No & $\%$ & № & $\%$ \\
\hline Almería & Médicos del mundo & Exclusión social & 2 & 3,8 & 45 & 2,6 & 2 & 4,9 & 49 & 2,7 \\
\hline Cádiz & $\begin{array}{l}\text { Comité Antisida } \\
\text { Campo de Gibraltar }\end{array}$ & VIH/Sida & 1 & 1,9 & 94 & 5,4 & 0 & 0,0 & 95 & 5,2 \\
\hline \multirow[t]{2}{*}{ Córdoba } & Cruz Roja & Exclusión social & 0 & 0,0 & 75 & 4,3 & 0 & 0,0 & 75 & 4,1 \\
\hline & Iemakaie & Exclusión social & 1 & 1,9 & 55 & 3,1 & 0 & 0,0 & 56 & 3,0 \\
\hline Huelva & Triángulo & LBGT & 1 & 1,9 & 64 & 3,7 & 1 & 2,4 & 66 & 3,6 \\
\hline \multirow[t]{2}{*}{ Málaga } & Concordia & VIH/Sida & 6 & 11,3 & 165 & 9,4 & 0 & 0,0 & 171 & 9,3 \\
\hline & Asima & VIH/Sida & 4 & 7,5 & 135 & 7,7 & 7 & 17,1 & 146 & 7,9 \\
\hline \multirow[t]{4}{*}{ Sevilla } & Adhara & VIH/Sida & 37 & 69,8 & 823 & 47,0 & 7 & 17,1 & 867 & 47,0 \\
\hline & Triángulo & LBGT & 0 & 0,0 & 169 & 9,7 & 20 & 48,8 & 189 & 10,2 \\
\hline & Médicos del mundo & Exclusión social & 1 & 1,9 & 125 & 7,1 & 4 & 9,8 & 130 & 7,0 \\
\hline & & Total & 53 & 100,0 & 1750 & 100,0 & 41 & 100,0 & 1844 & 100,0 \\
\hline
\end{tabular}

LGTB: lesbianas, gais, bisexuales y personas transgénero.

la prueba rápida de VIH y contestaron a la encuesta. Se analizó la información anónima recogida previamente a la realización de la prueba por parte de un encuestador, tras la obtención del consentimiento oral u escrito de la persona interesada, según las normas internas de cada entidad.

Se realizó un análisis descriptivo mediante medidas de tendencia central y dispersión para variables cuantitativas, y de frecuencia para las cualitativas. Para la comparación de las variables se realizaron la prueba de la t de Student, de la chi cuadrado $\left(\chi^{2}\right)$ de Pearson y la prueba exacta de Fisher con la determinación del grado de significación estadística bilateral correspondiente (p), utilizándose el paquete de programa estadístico SPSS v.19. Se calculó la Odds ratio, mediante tablas de contingencia, acompañadas del intervalo de confianza del 95\% (IC 95\%). Así mismo, se realizó un análisis multivariante de regresión logística, método paso a paso, incluyéndose aquellas variables más relevantes y significativas que se obtuvieron por $\mathrm{p}<0,10$. Una de las variables clave del estudio, la orientación sexual, se construyó a partir del sexo de la persona y de las relaciones mantenidas en los 12 meses previos a la consulta. Los varones se clasificaron como HSH si referían haber tenido relaciones sexuales con hombres u hombres y mujeres. Las mujeres no se clasificaron en función de sus relaciones sexuales. Para el estudio de los factores asociados a tener un resultado reactivo, se incluyeron todas las encuestas con un resultado reactivo independientemente de la realización de la prueba de confirmación.

\section{RESULTADOS}

Desde el inicio del Programa en diciembre de 2008 hasta diciembre de 2014, en Andalucía, se han realizado un total de 19.223 pruebas de detección rápida del VIH en saliva, con un total de 437 reactivos (2,3\%) (Fig. 1). Durante el periodo de estudio, en los 9 centros seleccionados, se encuestaron un total de 1.844 personas (el $48 \%$ del promedio anual de las pruebas realizadas en el bienio 2013-2014 en todas las entidades colaboradoras), con un total de 53 reactivos $(3,0 \%)$, y un $2,2 \%$ de resultados perdidos (Tabla 1). El 100\% (54) de 


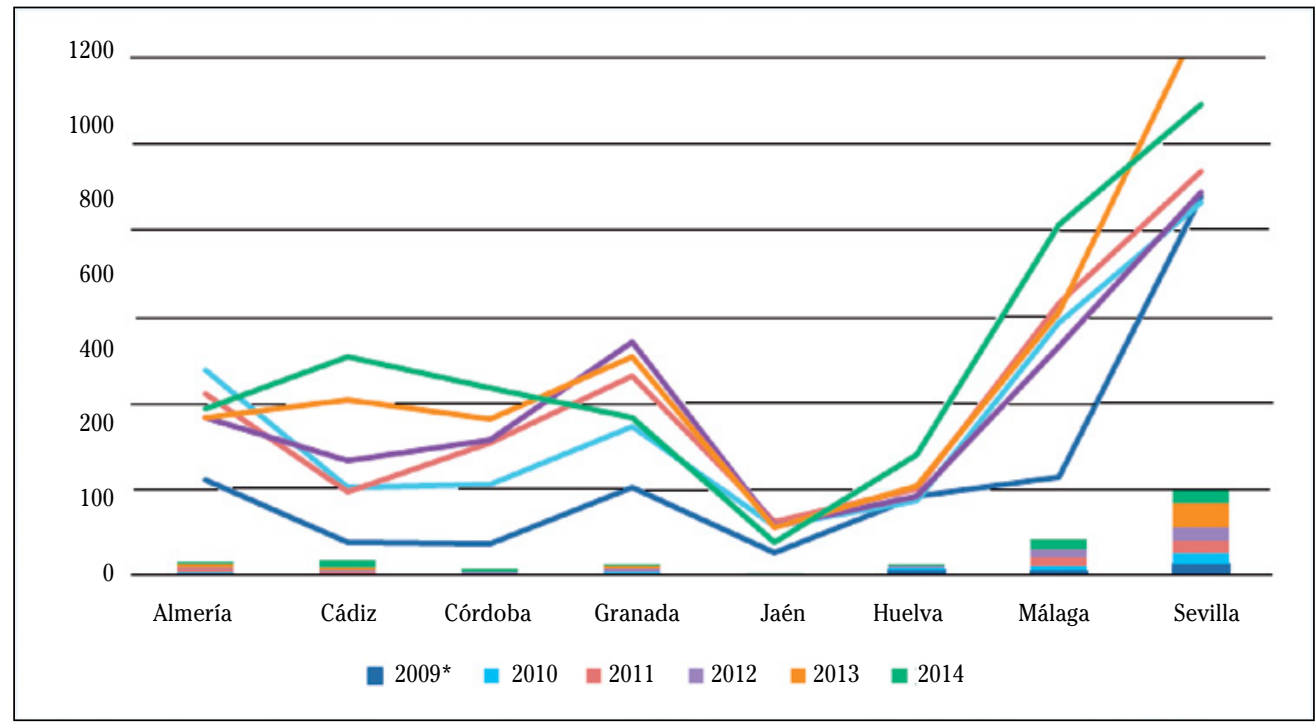

*Incluye diciembre 2008

Figura 1. Actividad en términos de número de pruebas (líneas) y reactivos (columnas apiladas) por año del Programa de diagnóstico precoz del VIH en Andalucía. Periodo 2009-2014.

los reactivos se derivaron a un centro hospitalario para la realización de una prueba de confirmación en sangre, sin embargo los resultados de la misma se recogieron solo en el 24,5\% (13) de los casos en los que se confirmó el diagnóstico.

Con respecto al perfil de las personas que demandaron la prueba, el $70 \%$ eran hombres. Entre ellos, el 59\% fueron $\mathrm{HSH}$. El $75 \%$ de los hombres acudió a entidades dedicadas a VIH/Sida frente al $60 \%$ de las mujeres $(\mathrm{p}<0,001)$. El $49 \%$ fueron menores de 30 años, el $44 \%$ entre los hombre y el $60 \%$ entre las mujeres $(\mathrm{p}<0,001)$. El $92 \%$ de las personas acudieron tras una exposición de riesgo, el resto sólo para control. La exposición de riesgo más frecuente fue haber mantenido relaciones sexuales desprotegidas de tipo oral (75\%), vaginal (50\%) y anal (38\%). El $19 \%$ refirió rotura del preservativo durante la exposición, el $8 \%$ sexo desprotegido con pareja $\mathrm{VIH}+$, el $4 \%$ sexo desprotegido con trabajador/a sexual, el 3\% otro tipo de riesgo. Solo el $0,5 \%$ de las personas compartió material de inyección. El 53\% de las personas se había realizado anteriormente una prueba de $\mathrm{VIH}$, el $51 \%$ de ellas en el último año. El $29 \%$ se había realizado previamente 4 o más pruebas. El $9 \%$ refirió entre sus antecedentes, un diagnóstico previo de infección de trasmisión sexual (ITS) en los últimos 12 meses. La mayoría de las personas que demandaron la prueba recibieron consejo por parte de un trabajador del centro, respectivamente antes (77\%) y/o después (64\%) de la misma, siendo elevado número de valores perdidos ( $23 \%$ y $35 \%$, respectivamente).

El 23\% fueron personas de nacionalidad extranjera que procedían de más de 50 países diferentes, siendo los más frecuentes Rumania (14\%), Colombia (11\%), Marruecos (9\%), Ecuador (7\%) y Brasil (5\%). El $47 \%$ acudió a entidades que se dedican a labores de exclusión social frente al $9 \%$ de los españoles $(\mathrm{p}<0,001)$. La mayoría fueron mujeres ( $77 \%$ vs $54 \%$ de los españoles, $\mathrm{p}<0,001$ ), el $43 \%$ fueron HSH frente al $64 \%$ de los HSH españoles $(\mathrm{p}<0,001)$. Un porcentaje significativamente mayor de extranjeros presentó antecedentes de prueba previas $(60 \%$ vs $52 \%$, $\mathrm{p}=0,01)$ y solo el $5 \%$ tenía antecedentes de diagnóstico de ITS en el último año (contra el 10\% de los españoles, $\mathrm{p}=0,01$ ), (Tabla 2). 
Tabla 2. Variables de estudio para la caracterización de las personas que demandaron la prueba de diagnóstico precoz del VIH. Periodo: septiembre 2013-agosto 2014

\begin{tabular}{|c|c|c|c|c|c|c|c|}
\hline \multirow{2}{*}{\multicolumn{2}{|c|}{ Val }} & \multicolumn{2}{|c|}{ Reactivos } & \multicolumn{2}{|c|}{ No Reactivos } & \multirow{2}{*}{ Valor P } & \multirow{2}{*}{$\begin{array}{l}\text { Odds ratio } \\
\text { (Intervalo de confianza del 95\%) }\end{array}$} \\
\hline & & № & $\%$ & № & $\%$ & & \\
\hline \multicolumn{8}{|l|}{ Labor entidades } \\
\hline VIH-Sida & & 48 & 90,6 & 1222 & 69,8 & \multirow[t]{2}{*}{$\mathrm{p}<0,001$} & \multirow{2}{*}{$\begin{array}{c}\text { VIHsida/OtroTipo OR= 4,15 (IC95\%= } \\
1,64-10,48)\end{array}$} \\
\hline Otro Tipo & & 5 & 9,4 & 528 & 30,2 & & \\
\hline \multicolumn{8}{|l|}{ Sexo } \\
\hline Hombre & & 48 & 90,6 & 1193 & 69,1 & \multirow[t]{2}{*}{$\mathrm{p}<0,001$} & \multirow{2}{*}{$\begin{array}{c}\text { Hombre/Mujeres OR }=4,30(\mathrm{IC} 95 \%= \\
1,70-10,88)\end{array}$} \\
\hline Mujeres & & 5 & 9,4 & 534 & 30,9 & & \\
\hline \multicolumn{8}{|c|}{ Práctica sexual entre los hombres } \\
\hline $\mathrm{HSH}$ & & 39 & 83,0 & 616 & 38,5 & \multirow[t]{2}{*}{$\mathrm{p}<0,001$} & \multirow{2}{*}{$\begin{array}{c}\mathrm{HSH} / \mathrm{NoHSH} \mathrm{OR}=7,79(\mathrm{IC} 95 \%=3,62- \\
16,79)\end{array}$} \\
\hline No HSH & & 8 & 17,0 & 985 & 61,5 & & \\
\hline \multicolumn{8}{|l|}{ Menores 30 años } \\
\hline Sí & & 22 & 41,5 & 837 & 48,9 & \multirow[t]{2}{*}{$\mathrm{p}=0,28$} & \multirow{2}{*}{$\begin{array}{c}\text { Sí/No OR }=0,74 \\
(\mathrm{IC} 95 \%=0,42-1,29)\end{array}$} \\
\hline No & & 31 & 58,5 & 873 & 51,1 & & \\
\hline \multicolumn{8}{|l|}{ Nacionalidad } \\
\hline Española & & 39 & 83,0 & 1198 & 76,9 & \multirow[t]{2}{*}{$\mathrm{p}=0,33$} & \multirow{2}{*}{$\begin{array}{l}\text { Española/Otra OR }=1,46 \\
(\text { IC95\%= } 0,68-3,16)\end{array}$} \\
\hline Otra & & 8 & 17,0 & 360 & 23,1 & & \\
\hline \multicolumn{8}{|c|}{ Motivos para realizar la prueba } \\
\hline Exposición de riesgo & & 49 & 92,5 & 1603 & 91,8 & \multirow[t]{2}{*}{$\mathrm{p}=1^{*}$} & \multirow{2}{*}{$\begin{array}{c}\text { Sí/No OR = 1,10 } \\
(\mathrm{IC} 95 \%=0,39-3,09)\end{array}$} \\
\hline Solo control & & 4 & 7,5 & 144 & 8,2 & & \\
\hline \multicolumn{8}{|c|}{ Exposición sexual desprotegida } \\
\hline \multirow[t]{2}{*}{ Vaginal } & $\begin{array}{r}\text { Sí } \\
\text { No }\end{array}$ & 5 & 71,4 & 720 & 82,2 & \multirow[t]{2}{*}{$\mathrm{p}=0,61^{* *}$} & \multirow[t]{2}{*}{$\begin{array}{c}\text { Sí/No OR }=0,54 \\
(\mathrm{IC} 95 \%=0,10-2,82)\end{array}$} \\
\hline & & 2 & 28,6 & 156 & 17,8 & & \\
\hline Anal & Sí & 33 & 67,3 & 605 & 37,5 & $\mathrm{p}<0,01$ & Sí/No OR = 3,44 \\
\hline & No & 16 & 32,7 & 1009 & 62,5 & & $(\mathrm{IC} 95 \%=1,88-6,30)$ \\
\hline Oral & Sí & 40 & 81,6 & 1203 & 74,5 & $\mathrm{p}=0,26$ & Sí/No OR = 1,52 \\
\hline & No & 9 & 18,4 & 411 & 25,5 & & $(\mathrm{IC} 95 \%=0,73-3,16)$ \\
\hline Rotura & Sí & 9 & 20,0 & 288 & 19,6 & $\mathrm{p}=0,94$ & Sí/No OR = 1,03 \\
\hline preservativo & No & 36 & 80,0 & 1188 & 80,4 & & (IC95\%= 0,49-1,16) \\
\hline Con trabajador/a & Sí & 5 & 10,9 & 50 & 3,7 & $\mathrm{p}=0,03^{*}$ & Sí/No OR = 3,134 \\
\hline sexo & No & 41 & 89,1 & 1285 & 96,3 & & (IC95\%= 1,19-8,27) \\
\hline Con persona & Sí & 13 & 27,1 & 96 & 7,0 & $\mathrm{p}<0,001$ & Sí/No OR = 4,94 \\
\hline VIH+ & No & 35 & 72,9 & 1276 & 93,0 & & $($ IC95\%= 2,53-9,64) \\
\hline Compartir material & Sí & 0 & 0 & 8 & 0,5 & $\mathrm{p}=1^{*}$ & ND \\
\hline droga & No & 49 & 100 & 1562 & 99,5 & & \\
\hline Otro tipo exposición & & 1 & 2,0 & 47 & 3,0 & $\mathrm{p}=1^{*}$ & Sí/No OR = 0,675 \\
\hline riesgo & No & 48 & 98,0 & 1569 & 97,0 & & (IC95\%= 0,09-4,99) \\
\hline
\end{tabular}




\begin{tabular}{|c|c|c|c|c|c|c|}
\hline \multirow{2}{*}{ Val } & \multicolumn{2}{|c|}{ Reactivos } & \multicolumn{2}{|c|}{ No Reactivos } & \multirow{2}{*}{ Valor P } & \multirow{2}{*}{$\begin{array}{c}\text { Odds ratio } \\
\text { (Intervalo de confianza del 95\%) }\end{array}$} \\
\hline & № & $\%$ & № & $\%$ & & \\
\hline \multicolumn{7}{|c|}{ Antecedentes Prueba VIH+ } \\
\hline Sí & 36 & 67,9 & 907 & 52,2 & \multirow[t]{2}{*}{$\mathrm{p}=0,02$} & \multirow{2}{*}{$\begin{array}{c}\text { Sí/No OR = 1,9 } \\
(\mathrm{IC} 95 \%=1,08-3,47)\end{array}$} \\
\hline No & 17 & 32,1 & 829 & 47,8 & & \\
\hline \multicolumn{7}{|c|}{ Antecedentes ITS últ.12 meses } \\
\hline Sí & 8 & 16,3 & 132 & 8,6 & \multirow[t]{2}{*}{$\mathrm{p}=0,06$} & \multirow{2}{*}{$\begin{array}{c}\text { Sí/No OR = 2,07 } \\
(\mathrm{IC} 95 \%=0,95-4,51)\end{array}$} \\
\hline No & 41 & 83,7 & 1401 & 91,4 & & \\
\hline
\end{tabular}

HSH: Hombres que practican sexo con otros hombres

*Exacto de Fisher (bilateral)

** Sólo se ha calculado por la población de No HSH (hombres y mujeres).

Con respecto a los factores asociados a un resultado reactivo, las entidades especializadas en $\mathrm{VIH} / \mathrm{Sida}$ detectaron un número significativamente mayor de reactivos $(\mathrm{p}<0,001)$. Los hombres concentraron el $91 \%$ de todos los reactivos y el sexo guardó relación significativa con el resultado de la prueba, siendo el riesgo más de 4 veces superior al de las mujeres $(p<0,01)$. Los HSH concentraron el $83 \%$ del total de las pruebas reactivas, el $11 \%$ se dieron en mujeres y el $6 \%$ en hombres no $\mathrm{HSH}$ $(\mathrm{p}<0,001)$. Los grupos de edad entre 25-29 y 30-34 años agruparon el $44 \%$ de los reactivos (24\% y $19 \%$ respectivamente) sin embargo la variable edad no guardó relación significativa con el resultado de la prueba. A pesar de las diferentes características de la población extranjera frente a la española, el país de procedencia no guardó relación significativa con el resultado de la prueba $(2,2 \%$ de reactivos en extranjeros vs $3,2 \%$ en españoles, $\mathrm{p}=0,33$ ). Practicar relaciones sexuales anales desprotegidas, haber tenido relaciones desprotegida con trabajador/a sexual o haber tenido relaciones desprotegidas con una persona $\mathrm{VIH}+$ fueron factores de riesgo para un resultado reactivo en la prueba de $\mathrm{VIH}(\mathrm{p}<0,05)$. Tener antecedentes de VIH se asoció a un riesgo doble en la obtención de un resultado reactivo $(\mathrm{p}=0,02)$. Se quedó al borde de la significación la relación entre antecedente de ITS y resultado reactivo de la prueba $(\mathrm{p}=0,06)$, (Tabla 2$)$.
Los resultados del análisis multivariante de regresión logística evidenciaron que los factores predictivos de un resultado reactivo fueron ser hombre, el haber practicado relaciones sexuales anales desprotegidas, el haber tenido relaciones desprotegidas con trabajador/a sexual y haber tenido relaciones desprotegidas con una persona $\mathrm{VIH}+$ (Tabla 3$)$.

\section{DISCUSIÓN}

El estudio llevado a cabo ha permitido caracterizar una parte importante (alrededor del 50\%) de la población diana del Programa de detección en fluido oral del VIH en Andalucía. Principalmente fueron hombres, de nacionalidad española, la mitad de los casos menores de 30 años. Según la práctica sexual, se trató más frecuentemente de personas $\mathrm{HSH}$, con antecedentes de prueba de $\mathrm{VIH}$, que acudieron tras una exposición considerada de riesgo, siendo la más frecuente el sexo oral desprotegido. La población extranjera presentó características diferentes a la española, siendo más frecuentemente mujeres (menos $\mathrm{HSH}$ entre los hombres), que realizaron la prueba en organizaciones principalmente de exclusión social. Los factores asociados a un resultado reactivo fueron sexo masculino, $\mathrm{HSH}$, realización de relaciones desprotegidas (anales, con persona $\mathrm{VIH}+\mathrm{y} / \mathrm{o}$ con trabajador/a del sexo) y antecedentes de 
Tabla 3. Resultados de la asociación entre las variables predictores incluidas en el modelo y el resultado reactivo de la prueba de diagnóstico precoz del VIH. Periodo: septiembre 2013-agosto 2014.

\begin{tabular}{lccc}
\hline \multicolumn{1}{c}{ Variables incluidas en el estudio } & Valor P & Odds ratio & IC 95\% \\
\hline Labor de la entidad VIH/Sida & 0,06 & 4,03 & $0,95-17,06$ \\
\hline Diagnóstico de ITS en los últimos 12 meses & 0,17 & 1,77 & $0,78-4,03$ \\
\hline Antecedentes de prueba previa & 0,78 & 1,00 & $0,57-2,13$ \\
\hline Realización de sexo desprotegido anal & 0,00 & 2,73 & $1,40-5,31$ \\
\hline Realización de sexo desprotegido con trabajador/a sexual & 0,02 & 3,41 & $1,28-9,43$ \\
\hline Realización de sexo desprotegido con persona VIH+sexo masculino & 0,00 & 4,42 & $2,13-9,19$ \\
\hline Constante & 0,03 & 4,82 & $1,12-20,73$ \\
\hline
\end{tabular}

ITS: Infección de trasmisión sexual

prueba de VIH y haber realizado la prueba en entidades especializadas en $\mathrm{VIH} /$ sida.

Esta evidencia es el fruto de la gran labor realizada por las entidades en Andalucía que, junto con otras entidades a nivel nacional y a las farmacias comunitarias, están colaborando muy activamente en los diferentes programas de detección rápida del $\mathrm{VIH}^{12-13}$. Otras experiencias, algunas de menor calado o con un carácter piloto, se están llevando a cabo en centros sanitarios (Atención Primaria, Atención Especializada y Unidades de ITS), universitarios y redes privadas de laboratorios ${ }^{14-17}$. Concretamente, los centros de salud constituyen el primer punto de contacto de la población con el sistema sanitario, y son claves para la intensificación de la oferta de la prueba de VIH en Atención Primaria, siendo una de las estrategias prioritarias para mejorar el pronóstico de los pacientes con infección por $\mathrm{VIH}^{10,18}$.

En al ámbito de las entidades sociales, existe una realidad fragmentada con una variedad de programas en la que destaca el Proyecto europeo HIV-Cobatest o el gestionado por BCN Checkpoint ${ }^{12-13}$, que cuenta con la colaboración de entidades orientadas a diferentes tipo de colectivos vulnerables (lesbianas, gais, bisexuales y personas transgénero-LGTB, inmigrantes, mujeres que ejercen la prostitución, exclusión social, etc.).

Los resultados obtenidos en nuestro estudio están en línea con otros desarrollados por las entidades sociales con respec- to al enfoque hacia una población joven, las mujeres más que los hombres, el abordaje de personas con prácticas sexuales de riesgo y con antecedentes de ITS $^{19-20}$. Sin embargo, se obtuvieron menores porcentajes de mujeres y extranjeros, y mayores porcentajes de $\mathrm{HSH}^{21-22}$. Estas diferencias podrían explicarse por el gran número de pruebas realizadas por entidades especializadas en $\mathrm{VIH} /$ sida de nuestro estudio por el tipo de población atendida.

En relación a la oferta de pruebas rápidas en las farmacias comunitarias, desde 2009, se han documentado experiencias en diferentes comunidades, destacando las del País Vasco, Cataluña, Castilla y León ${ }^{23-24}$. En comparación con la población diana de las farmacias, las personas caracterizadas en nuestro estudio son más jóvenes, en mayor porcentaje extranjeros, principalmente de sexo masculino y HSH. Sin embargo, ambos tipos de programas, permiten alcanzar a una población sin antecedentes de haber realizado prueba de VIH anteriormente que ronda el 50\%. El porcentaje global de resultados reactivos de estos programas es sustancialmente menor (0,8-1,3\%) en comparación con aquella de los programas dirigidos a poblaciones de riesgo (alrededor del $3 \%)$.

El Programa de detección rápida de VIH en Andalucía, como otros programas de este tipo, oferta una prueba con mayor aceptación respecto a las pruebas convencionales $^{25}$ y propicia estrategias de captación activa entre colectivos vulnerables ${ }^{26}$. 
Además, fomenta la posibilidad de realizar una labor específica de counselling y acompañamiento, especialmente valiosa en el caso de las personas con resultados reacti$\operatorname{vos}^{27}$ y la oportunidad de alcanzar a personas con posibles barreras de acceso al sistema sanitario. La aprobación de la venta a la población general del autotest doméstico, la comercialización de nuevos dispositivos que permiten acortar el periodo ventana y de las profilaxis de pre-exposición previsiblemente tendrán un impacto relevante en los programas de prueba rápida de VIH existentes en la actualidad ${ }^{12,28-29}$. Por ello, es importante seguir en la implementación de esta prueba e ir adaptando el Programa. En esta línea, en Andalucía, se está trabajando en el desarrollo de procedimientos de recogida de datos y herramientas informáticas que permitan disponer de una información más fiable y completa acerca de la población atendida por todas las entidades del Programa, y, paralelamente, reforzar un enfoque de convergencia hacia la creación de una Red de Programas Comunitarios de Diagnóstico de VIH (RedCo-VIH) ${ }^{30}$.

En relación a las limitaciones del estudio, hay que destacar que no se pudo analizar la información de todas las entidades de Andalucía debido al carácter voluntario de la colaboración solicitada y al escaso interés epidemiológico de las encuestas utilizadas por algunas de estas entidades. Además, se incluyeron en el análisis solo aquellas variables recogidas por todas las entidades seleccionadas.

Desde su implantación el Programa de diagnóstico precoz del VIH de Andalucía ha permitido detectar un gran número de personas infectadas haciendo de la prueba rápida una herramienta fundamental de prevención en colectivos vulnerables. Los resultados del presente estudio aportan evidencia sobre las principales características y factores de riesgo de un elevado número de personas (alrededor del 50\%) que demandaron la prueba de detección precoz de VIH. A corto plazo, favorecerán una mejor planificación de las necesidades de dispositivos para la detección del VIH y la definición de procedimientos de recogida de datos adaptados al perfil de las per- sonas y a las necesidades de las entidades colaboradoras del Programa, siendo esta última un área de mejora prioritaria.

\section{Agradecimientos}

A todas las entidades colaboradoras del Programa de detección rápida de VIH en Andalucía y especialmente a: Adhara, Asima, Concordia, Fundación Triángulo, Iemakaie y Médicos del Mundo. A Juan Serrano Lara y Maria Jesús Lopez Fernández por su imprescindible colaboración.

\section{BIBLIOGRAFÍA}

1. Marks G, Crepaz N, Janssen RS. Estimating sexual transmission of HIV from persons aware and unaware that they are infected with the virus in the USA. AIDS 2006; 20: 1447-1450.

2. Alastrué I, Díaz F, Santos F, Corrons AJ. ¿Son efectivos los centros específicos de VIH/ITS para disminuir el retraso del diagnóstico en la infección por el virus de la inmunodeficiencia humana? Enferm Infecc Microbiol Clin 2014; 32: 689-696.

3. Área de Vigilancia de VIH y Comportamientos de Riesgo. Vigilancia Epidemiológica del VIH y sida en España: Sistema de Información sobre Nuevos Diagnósticos de VIH y Registro Nacional de Casos de Sida. Plan Nacional sobre el Sida [Informes de actualización]. Madrid: ISCIII, nov 2015; [consultado 19-12-2015]: Disponible en: http://www. msssi.gob.es/ciudadanos/enfLesiones/enfTransmisibles/sida/vigilancia/home.htm

4. Sistema Vigilancia epidemiológica de Andalucía. Nuevos diagnósticos de infección por el VIH y sida notificados desde enero 2013. Evolución temporal de sida en Andalucía desde 1982. Actualización a 26 de junio de 2015. Sevilla: Consejería de salud de Andalucía, 2016

5. Rodríguez M, Zamora C. Plan Andaluz frente al $\mathrm{VIH} /$ sida y otras ITS 2010-2015. Sevilla: Consejería de Salud de Andalucía, 2010

6. Zelin J, Garrett N, Saunders J, Warburton F, ANDERSON J, Moir K et al. An evaluation of the performance of OraQuick ADVANCE Rapid HIV-1/2 Test in a high-risk population attending genitourinary medicine clinics in East London, UK. Int J STD AIDS 2008; 19: 665-667

7. Zachary D, Mwenge L, Muyoyeta M, Shanaube K, SchaAp A, Bond V et al. Field comparison of 
OralQuick ADVANCE Rapid HIV-1/2 antibody test and two blood-based rapid HIV antibody tests in Zambia. BMC Infect Dis 2012; 12: 183

8. Oliva G, Almaź́n M, Guillén M. Prueba de detección rápida de la infección por VIH [Informes de Evaluación de Tecnologías Sanitarias, AATRM núm. 2007/03]. Madrid: Plan de Calidad para el Sistema Nacional de Salud. Ministerio de Sanidad y Política Social. Agència d'Avaluació de Tecnologia i Recerca Mèdiques de Cataluña; 2009; [consultado 1509-2015]: Disponible en: http://aunets.isciii. es/ficherosproductos/sinproyecto/602_aatrm2007-03.pdf

9. European Centre for Disease Prevention and Control, WHO Regional Office for Europe. HIV/AIDS surveillance in Europe 2014. Stockholm: ECDC, 2015.

10. Ministerio de Sanidad, Servicios Sociales e Igualdad. Guía de recomendaciones para el diagnóstico precoz del VIH en el ámbito sanitario. Madrid: MSSSI, 2014.

11. Broeckaert L, Challacombe L. Rapid point-ofcare HIV testing: a review of the evidence. Toronto: Canada's source for HIV and hepatitis C Information (CATIE), 2015; [consultado 15-10-2015]: Disponible en: http://www.catie. ca/en/printpdf/pif/spring-2015/rapid-pointcare-hiv-testing-review-evidence

12. Arrillaga A, Zulaika D. Situación actual de los test rápidos de VIH en nuestro país. Revista Multidisplinar del Sida 2015; 3: 57-66.

13. Reyes-Urueña J, Breveglieri M, Furegato M, FernÀndez-López L, Agusti C, Casabona J et al. Heterogeneity of community-based voluntary, counselling and testing services for HIV in Europe: the HIV-COBATEST survey. Int J STD AIDS 2015; 0: 1-11.

14. Gorostiza I, Elizondo López de Landache I, BraCERAs IZAGIRRE L. Programa de cribado de VIH/ sida en las oficinas de farmacia en la Comunidad Autónoma del País Vasco. Gac Sanit 2013; 27: 164-166.

15. Cuesta MM, López MC, Nieto P, Junquera ML, VARELA JA, VASQUEZ $F$ et al. Implantación de una prueba rápida de VIH en Asturias (España). Enferm Infecc Microbiol Clin 2012; 30: 189-191.

16. Moreno S, Ordobás M, Sanz JC, Ramos B, Astray J, Ortiz M et al. Prevalence o fundiagnosed HIV infection in the general population having blood tests within primary care in Madrid, Spain. Sex Transm Infect 2012; 88: 522-524.

17. Hoyos J, de la Fuente L, Fernández S, Gutiérrez J, Rosales ME, García de Olalla P et al. Grupo de Pruebas Rápidas de VIH Madrid. La oferta de la prueba rápida del VIH en la calle den- tro del ámbito universitario: ¿una estrategia prioritaria? Gac Sanit 2012; 26: 131-137.

18. Martín-Cabo R, Losa-García JE, Iglesias-Franco $\mathrm{H}$, Iglesias-GonzÁlez R, Fajardo-Alcántara A, JimÉnez-Moreno A et al. Promoción de la detección del virus de la inmunodeficiencia humana en atención primaria. Gac Sanit 2012; 26 (Suppl 2): 116-122.

19. Marcus U, Ort J, Grenz M, Eckstein K, Wirtz K, WILLE A et al. risk factors for HIV and STI diagnosis in a community-based HIV/STI testing and counselling site for men having sex with men (MSM) in a large German city in 20112012. BMC Infect Dis 2015; 15:14.

20. Diez M, Bleda MJ, Varela JA, Ordoñana JR, AzPIRI MA, VAll M et al. Trends in HIV testing, prevalence among first-time testers, and incidence in most-at-risk populations in Spain: the EPI-VIH Study, 2000 to 2009. Euro Surveill 2014; 19 (Suppl 47): pii=20971

21. Belza MJ, Hoyos J, Fernández-Balbuena S, Diaz A, Bravo MJ, dE la Fuente L et al. The Madrid HIV rapid testing group. Assessment of an outreach street-based HIV rapid testing programme as a strategy to promote early diagnosis: a comparison with two surveillance systems in Spain, 2008-2011. Euro Surveill 2015; 20 (Suppl 14): pii=21089.

22. Hoyos J, Belza MJ, Fernández-Balbuena S, Rosales-Statkus ME, Pulido J, DE la Fuente L et al. Preferred HIV testing services and programme characteristics among clients of a rapid HIV testing programme. BMC public health 2013; 3: 1-9.

23. Fernández-Balbuena S, Belza MJ, Zulaica D, Martinez JL, Marcos H, Rifá B et al. Widening the Access to HIV Testing: The Contribution of Three In-Pharmacy Testing Programmes in Spain. PLoS ONE [revista electrónica] 2015; 10(8): e0134631 [consultado 15-09-2015]: Disponible en: http://journals.plos.org/plosone/ article?id=10.1371/journal.pone.0134631

24. Fernandez-Balbuena S, Marcos H, Perez-Rubio A, Hoyos J, Belza MJ, de la Fuente L et al. The rapid test in Spanish pharmacies: a novel programme to reach heterosexual men? HIV Med 2015; 16 (Suppl 6): 362-369.

25. Read TRH, Hocking JS, Bradshaw CS, Morrow A, GRulich AE, Fairley CK et al. Provision of rapid HIV tests within a health service and frequency of HIV testing among men who have sex with men: randomied controlled trial. Brit Med J 2013; 347: 5086.

26. Turner SD, Anderson K, Slater M, Quigley L, Dyck M, Guiang CB. Rapid point-of-care HIV testing in youth: A systematic review. J Adolesc Health 2013; 53: 683-691. 
27. Roberts KJ, Grusky O, Swanson A-N. Outcomes of blood and oral fluid rapid HIV testing: A literature review, 2000-2006. AIDS Patient Care and STDs 2007; 21: 621-637.

28. Thigpen MC, Kebaabetswe PM, Paxton LA, Smith DK, Rose CE, SEgolodi TM et al. TDF2 Study Group. Antiretroviral preexposure prophylaxis for heterosexual HIV transmission in Botswana. N Engl J Med 2012; 367: 423434.
29. Baeten JM, Donnell D, Ndase P, Mugo NR, Campbell JD, WANGISIJ et al. Antiretroviral prophylaxis for HIV prevention in heterosexual men and women. N Engl J Med 2012; 367: 399-410.

30. World Health Organization Scaling up HIV testing and counseling in the WHO European Region: as an essential component of efforts to achieve universal access to HIV prevention, treatment, care and support. Policy framework. Geneva: WHO, 2010. 